\title{
Author index to Volume 83
}

\section{Key to abbreviations:}

(BR) Book reviews; (L) Letters to the editors

Aaltonen L 1643

Aaltonen LA 1015

Aapro MS 1637

Aasheim H-C 743

Abbou CC 209

Abdulkarim B 514

Abe T 887

Abecassis J 1380

Abels C 1216

Abra RM 232, 684

Actane Consortium 1654

Adami H-O 949

Adams J 561

Agudo A 104

Ahern R 980

Aherne GW 146, 792

Ahlbom A 692

Ahmed A 127

Ahmed T 360

Aikou T 701

Akagi K 887

Akahira J 1488

Akazawa K 986

Akedo H 769

Akhmedkhanov AA 1255 (L)

Akinaga S 1039

Akisik E 737

Alberto P 1637

Albo D 298

Albright RE 588

Alemi M 307

Alexander FE 487

Alexopoulos CG 1128

Alho O-P 614

Ali SA 1061

Allal AS 1637

Allen DG 1659

Allen MJ 980

Allen NE 95

Allman R 655

Altomonte V 1405

Altorki N 493

Alvarez B 526

Amato R 1256 (L)

Amino N 1495

Amir Z 284

Andersen TI 1650

Anderson DQ 1061

Anderson E 992

Anderson EDC 98

Anderson H 447

Anderson JJ 498

Anderson TJ 487

Andersson S 307

Andoh A 769

Andrion A 104
Andrulis IL 737

Angeletti CA 480

Anglian Breast Cancer Study

Group 1301

Angus B 498

Anselmi L 1412

Anthoney A 426

Anthuber M 1216

Appleby PN 95

Aquino-Parsons C 1525

Araki K 609

Araki T 196

Arcangeli A 1722

Archimandritis S 1281

Arends J-W 252

Argilés JM 526

Aridome K 701

Armatis P 906

Armes JE 1659

Armstrong DK 1405

Ascierto PA 1707

Ashley S 91, 1418

Asselain B 1480

Atzpodien J 583

Auclerc M-F 1617

Aurlien E 1375

Austrup F 1664

Autier P 1243

Auvinen A 956

Awad L 431

Awwad HK 30

Ayude D 1139

Baba H 141, 1168

Baccino FM 526

Bachellerie-Rhein 1594

Bader S 1646

Bailey D 421

Baird MS 914

Balkwill FR 1538

Baniel J 463

Banks E 412

Barak M 1696

Barber MD 1443

Barberi-Heyob M 1380

Barbet N 594

Barbier P 544

Barbone F 1238

Barfoot R 177

Baron PL 16

Baronius W 458

Barraclough R 1084, 1473

Barret J-M 1740

Barth S 252

Baruchel A 1617

Baskaran R 1360
Basolo F 480

Bastuji-Garin S 209

Bate S 980

Bates SE 817

Baudin E 715

Baum M 1769 (BR)

Baumann P 1387

Baumöhl J 1128

Bax CMR 1730

Bay BH 319

Becchetti A 1722

Begg AC 899

Beijnen J 375

Beketic-Oreskovic L 892

Belometti MO 573

Belzile E 112

Ben-Eliyahu S 1630, 1747

Benasso M 1437

Benedict WF 1039

Benepal TS 146

Bengisu E 737

Beretta GD 573

Bergenheim AT 826

Bergerat J-P 346

Bergeron C 973

Berghmans T 1128

Bergman L 1003, 1009

Bergström R 391

Berner A 743

Berney CR 1637

Berrington A 412

Berry C 1047

Besret C 1309

Beuzeboc P 1480

Bicknell R 63

Bicocchi MP 1295

Bilbao J 847

Binns W 1565

Birch JM 467, 1136

Birchall MA 421

Bischoff P 642

Bishop MC 443

Bissery MC 544

Bitisik O 737

Bjellerup P 171

Black RJ 387

Blagosklonny MV 817

Blake C 1330

Blakey DC 267

Bleiberg H 431

Blok LJ 246

Böckmann B 1664

Boehm J 473

Boivin J-F 112

Bokemeyer C 458

Boldrini L 480
Bolger B 566

Bombardelli E 1762

Bonaïti-Pellié C 177

Bond MG 447

Bond S 1405

Boothroyd C 1003

Bordigoni P 1617

Borg A 775

Borrani E 1722

Børresen-Dale AL 1650

Borri P 1722

Borst P 366, 375

Bosetti C 689

Bosserhoff A-K 1216

Botti G 1707

Botting B 278 (L)

Bouffler SD 1291

Bourhis J 514

Boutard P 973

Boven E 921

Bower M 1432

Bowman A 98

Bown NP 40

Bowskill C 935

Boyano MD 847

Boyer M 438

Boyle JM 467, 1136

Brada M 588

Bradshaw TD 270

Brannan M 1599

Brattsand G 311

Braybrooke JP 219

Breitkreutz D 1387

Brekelmans CTM 384

Brewster DH 387

Bright E 1405

Brinkmann AO 246

Britton JA 1552

Brodie AM 74

Bromelow KV 853

Broome P 443

Brossault S 1418

Brown JE 1448

Brown KW 1583

Brown NJ 1061

Bruchim Bar Sade R 153

Bruland ØS 1375

Bruner J 588

Buccoliero AM 1722

Buer J 583

Buettner R 1216

Bugat R 431

Burchell J 1202

Burt EC 467

Burt PA 447

Busam KJ 493 
Busch R 35

Bush JA 1468

Busquets S 526

Bussink J 674

Butow PN 1448

Bykovskaia SN 506

Cabioglu N 737

Cagini L 569

Cai Q 506

Cailleux AF 715

Calcinai A 480

Caldas C 1309

Calderwood MA 329

Calleja A 104

Calogero A 184

Calsou P 514

Calvete JA 267

Cameron D 1009

Cameron DA 98

Cameron DW 134 (L)

Campbell MJ 959

Cañavate ML 847

Capdeville R 594

Cappello M 569

Carbó N 526

Cardinal J 1009

Cardinali B 1503

Carestia L 8

Carmichael J 447

Carothers A 1643

Carozzi F 1462

Carpi A 1412

Carthew P 935

Castello G 1707

Castiglione F 707

Casula M 1707

Cazenave J-P 346

Cerignoli F 1503

Césarini J-P 1243

Chadha-Ajwani S 246

Chamoun E 1243

Chang A 750

Chaplin DJ 811

Chapman AD 632

Chard T 1730

Chastagner P 973

Chatzaki E 1730

Chatzistamou I 906

Cheeseman SL 1599

Chellini E 104

Chen C-L 1096, 1674

Chen GK 892

Chen J-Y 1674

Chen Y-T 493

Chen Z 1367

Cheng A-L 1510

Cheng KK 127

Cherian SP 83

Cherubini A 1722

Cheung Jr K-JJ 1468

Chia S-J 761

Chiao JW 360

Chilvers CED 127

Chin K-F 1425

Chin S-F 1309

Chisamore MJ 782

Choi L 594

Chompret A 177

Chopin DK 209
Chott A 454

Chow VTK 319

Christen RD 1360

Christmas TJ 1274, 1432

Christofori G 1

Chua M-S 270

Ciatto S 1462

Cigolari S 707

Ciolino HP 333

Clark IM 1147

Clarke B 1405

Clarke NW 992

Clarke RB 992

Clemens M 458

Coates AS 1448

Coche-Dequeant B 1594

Cocker HA 338

Coelho D 642

Cole DJ 16

Collet J-P 112

Collins N 83

Colombino M 1707

Colpaert F 1516

Colston KW 239

Compagni A 1

Confortini M 1462

Connolly CK 447

Conte M 1412

Cook-Mozaffari P 127

Cooke T 566

Cooper GS 404

Cooper RA 620

Coplan K 493

Coralli C 662

Cordero OJ 1139

Cornelisse CJ 719

Cornu G 1617

Cortés RE 969 (L)

Corvò R 1437

Cossu A 1707

Costelli P 526

Cotterill SJ 397

Cottier B 447

Couillault G 973

Courtay-Cahen C 1309

Courtney M 1454

Cowburn P 655

Cox R 1291

Coze C 973

CPT-11 F205, F220, F221 and

V222 study groups 431

Craft AW 397

Crociani O 1722

Croke DT 725

Crowl-Bancroft CV 1367

Csánady M 261

Cui X 50

Culine S 431

Culjak G 1448

Cullen MH 1623

Cunningham D 146

Cunningham DC 287

Curtin NJ 267

Cuzick J 561

Dachs GU 662

Dagan E 153

Dalay N 737

Daley FM 30

Dalgleish AG 239
Dalmasso P 104

D'Amico M 1437

Danesh J 970 (L)

Darbre PD 1183

Darby S 412

Daurès J-P 8

Davey GK 95

David K 1747

David S 544

Davidson JM 1309

Davidson NE 1405

Davidson SE 620, 1702

Davies P 561

Davies S 1730

Davis JM 1405

Day N 121, 692

Day NE 127

de Bock GH 719

de Bono JS 426

de Haas M 366, 375

De Raucourt D 1594

de Rooij FWM 539

De Stavola BL 964

de Tourreil S 177

de Vries EGE 594

de Wilde PCM 674

de Wilt JHW 1176

Deacon JM 1565

Dearnaley DP 1623

Defachelle AS 973

Del Governatore M 1544

del Mar Melero-Montes M 1556

Deming SL 1688

Denhardt DT 156

Deo H 412

Deplanque G 346

Derby LE 1556

Desal M 1565

Deutsch E 514

Devalck C 973

Devilee P 719

Dhanabal M 1077

Di Marcotullio L 1503

Dias S 1538

Díaz-Pérez JL 847

Díaz-Ramón L 847

Dickens D 1599

Dickson RB 1688

Dictor M 775

Didelot C 1380

Diéras V 1480

Dincer M 737

Dinsdale D 935

Distefano M 1762

Dockerty J 692

Doherty AP 1432

Domenge C 1594

Donadieu J 1617

Dong C 407

Dong G 1367

Donnelly CA 134 (L)

Doong S-L 1674

Doré J-F 1243

Dorval T 1454, 1480

dos Santos Silva I 964

Doweck I 1696

Dragosics B 454

Driesel G 1664

Droz J-P 431

Duffey DC 1367
Duffy S 121

Dufour P 642

Dumez H 594

Dunican D 725

Dunlop MG 1643

Dunn SM 1448

Durán GE 892

Durand RE 1525

Dürmüller U 204

Duthie SJ 1532

East SJ 267

Eder C 1664

Edixhoven A 539

Edwards DR 1147

Edwards PAW 1309

Eggermont AMM 6, 1176, 1351

Einatan J 761

Eisen T 980

Ekbom A 949

Ekström AM 391

Ekström TJ 1020

Ellershaw C 1124

Elsässer-Beile U 637

Engelstein D 463

Engert A 252

English MA 550

EORTC Melanoma Co-operative Group 1243

Erikstein B 1650

Esche C 506

Eschwege F 514

Escolar A 104

Esmailzadeh A 1249

Espié M 577

Etiévant C 1516

Eu K-W 750

European Organization for

Research and Treatment of

Cancer 1351

Evans CD 1565

Evers R 366, 375

Extra J-M 1480

Fahimi S 1249

Falk SJ 447

Farahvash MJ 1249

Farmery SM 188

Farrington SM 1643

Farris A 707

Faulkner SW 729

Fearon KCH 1443

Felletti R 707

Fellous A 544

Fenwick F 498

Ferguson JSJ 1268

Ferlini C 1762

Ferrari P 1412

Ferrier CM 1351

Fetting J 1405

Feychting M 692

Fezoulidis I 1281

Field WN 56

Figer A 153

Fink K 588

Fink U 473

Floderus B 1231

Florin MC 1128

Floris C 1707

Flux GD 287 
Fodde R 1291

Fontana L 1722

Fontanini G 480

Foote D 278 (L)

Ford HER 146

Formanek M 454

Forouhi P 98

Forrest APM 487

Fortier I 1559

Fosså A 743

Fosså SD 743, 1623

Foulkes W 177

Fourquet A 1318

FRALLE Group 1617

Franceschi S 689, 1238

Franzke A 583

Fraser LA 387

Frater A 561

Frati L 1503

Fredericks R 588

French Acute Lymphoblastic Leukaemia (FRALLE) Group 1617

French Group d'Etude des Tumeurs de la Tête et du Cou (GETTEC) 1594

Freneaux P 1318

Frey M 1338

Freyer G 431

Fridman WH 1454

Friedlander ML 729

Friedman E 153, 463

Friedman H 588

Frisch M 89

Fritsche H 1256 (L)

Frontini L 707

Fujii H 1607

Fukuda R 1394

Fukuda T 1168

Futamura N 1681

Gallagher CJ 1612

Gallo C 707

Gammon MD 1552

Ganesan TS 219

Gao F 699 (L)

García de Galdeano A 847

García-Vázquez MD 847

Gardeazabal J 847

Gardiner E 1425

Gardner C 1599

Garland SM 1659

Gasparini G 707

Gaudernack G 743

Geloen A 1055

Gem Vin Investigators 707

George NJR 992

George SL 959

Gershoni-Baruch R 153

Gescher A 935

GETTEC 1594

Ghazizadeh M 196

Giaccone G 1069

Giachi M 1722

Giannini G 1503

Giesing M 1664

Gil-Diez de Medina S 209

Giles MS 329

Giokas G 1234

Glantz M 588
Glendon G 737

Goepel JR 1061

Goh H-S 761

Goldstraw P 569

González CA 104

Goodwin DJ 329

Goodwin S 519

Gordon RJ 22

Gore ME 980

Gorman T 1084

Gornall P 602

Gorringe KL 1309

Goto S 1096

Gounon P 544

Graham J 426

Grant G 1532

Gray SG 1020

Grdina W 782

Greaves MJ 467, 1136

Green J 412

Greenberg E 1696

Greenberg H 588

Greenman J 1425

Gregory K 853

Gregory WM 98

Gridelli C 707

Grigoryev DN 74

Grill H-J 1664

Grimmond S 1003

Gritti G 573

Grivegnée A 1243

Groen HJM 594

Grondin A 998

Grothey A 870

Groupe d'Etude des Tumeurs à

Calcitonine 715

Groves F 956

Gruia G 146

Grussenmeyer T 637

Guarneri D 1437

Guba M 1216

Gudat F 204

Guillou PJ 188

Gulino A 1503

Gulisano M 707

Gumbrell L 1599

Gurney H 438

Haag C 458

Hain SF 863

Hakulinen T 956

Hall KT 329

Hamblin MR 1544

Hamilton G 454

Han EK-H 83

Hanley JA 112

Hansson L-E 391

Harani N 941

Hardcastle A 792

Hardy R 964

Harland SJ 1623

Harper PG 863

Harrington KJ 232, 684

Harris AL 63, 219

Harrison D 1646

Hart SL 662

Hartley L 1003

Hartmann JT 458

Hartmann O 973

Harvey P 1147
Hasan T 1544

Hasegawa Y 1495

Hashizume R 870

Haslett C 941

Hatano K 324

Haugk B 498

Haustermans K 899

Haward RA 284

Hayashi H 1209

Hayes AJ 1154

Hayflick L 841

Hayward N 1003, 1009

Hazama S 1026, 1209

Heberer M 204

Helmerhorst TJM 246

Hemminki K 407

Hempel V 458

Hendry JH 1702

Henriksson R 826

Henry-Amar M 577

Herait P 431

Hernandez S 104

Héron J-F 577

Herrero R 1238

Herrmann R 1454

Hess AD 1405

Hewison M 550

Higashiyama T 1495

Hilinski J 1047

Hill BT 1516, 1740

Hill C 1594, 1617

Hill SA 811

Hinnen P 539

Hiraoka S 668

Hirose H 1681

Hirose K 1026, 1209

Hjelm NM 1330

Ho CS 1330

Ho SKW 1330

Hoelscher AH 473

Hoffmann R 583

Hofland I 899

Hofland N 1291

Hohl RJ 588

Hohmann B 756

Holder AL 811

Holdsworth H 1538

Hole D 566

Holl V 642

Holm NV 1231

Hoogenboom HR 252

Hoos A 1323

Hopster D 1287

Hopwood P 447

Horikoshi N 141

Horn T 1405

Horne CHW 498

Horwich A 1623

Hospers GAP 184

Houlbrook S 219

House A 1261

Howe GR 1552

Howell SB 1047

Hoznek A 209

Hsieh C-c 1255 (L)

Hsu C-H 1510

Huang W-Q 1154

Huddart RA 863

Hughes SV 550

Hui A-M 50
Huikeshoven FJ 246

Hulscher TM 921

Humphreys JE 443

Hunai H 769

Hunter RD 620

Hussey HJ 56

Hutchins A-M 1659

Hyrynkangs K 614

Ianniello GP 707

Ichinose Y 928

Ide M 1607

Iizuka N 1026, 1209

Ikeda Y 887

Ikematsu S 701

Iles RK 1730

Illsley MC 650

Imbert T 1516

Imeson J 602, 1124

Inagaki N 56

Infante-Rivard C 1559

Inoue T 1488

Ishiguro S 769

Ishihara S 1394

Ishitsuka H 56

Ito K 1488

Ivaldi C 104

Iversen K 493

Izu R 847

Jäckel S 1664

Jackman AL 792

Jackson SP 1702

Jacobs E 443

Jacobs I 279 (L)

Jacobs IJ 1287

James LA 467

Jantscheff P 1454

Janulis L 519

Jauch K-W 1216

Jaurand M-C 1147

Jia W 1468

Jick H 1556

Jin E 196

Jin R 319

Johansen C 89

Johansson M 826

Johnson JI 1401

Johnson PJ 1330

Johnsson A 1047

Johnston S 980

Joiner M 514

Jokinen K 614

Joly F 577

Jónasson JG 1715

Jones J 1344

Jones M 1084

Jordan VC 782

Jorna AS 1176

Jörnvall H 171

Jouve M 1480

Joyeux I 1454

Juang B-T 1674

Julious SA 959

Jung R 874

Jungbluth AA 493

Kaanders JHAM 674

Kadomatsu K 701

Kadouri E 153 
Kahlos K 880

Kaibuchi K 1168

Kakeji Y 986

Kambouchner M 1318

Kameyama M 769

Kanayama S 668

Kancherla R 360

Kane KF 550

Kannagi R 1681

Kantola S 614

Kanz L 458

Kåresen R 1650

Karkavitsas N 1281

Karpus WJ 519

Kasinetz L 153

Kawaguchi H 609

Kawanami O 196

Kawano K 1096

Kawashima K 1394

Kaye JA 1556

Kaye SB 426

Kazumori H 1394

Keilholz U 6

Kelekis N 1281

Kelland LR 338

Kelleher DK 225

Kelsey A 602

Kennard DA 853

Kennedy MJ 1405

Kern FG 1154

Kerr KM 632

Keshoofy M 1249

Keshtgar M 1769 (BR)

Key TJ 95

Khan P 1599

Khuder SA 133 (L)

Kijima H 833

Kikuchi M 701

Kikuchi R 215

Kim CJ 1503

Kimura H 858

Kinnula V 880

Kinoshita J 887

Kinoshita Y 1394

Kirchner H 583

Kirk D 426

Kitamura K 609

Kitamura S 668

Kitano S 1096

Kitayama J 324

Kiviniemi M 1161

Kiyohara T 668

Kjartansson J 1715

Klamut HJ 998

Klastersky J 1128

Kleeberg UR 1351

Klein-Soyer C 346

Klijn JGM 384

Klimka A 252

Ko K 1039

Kobune-Fujiwara Y 769

Kocher T 204

Kogner P 171

Kohler JA 1124

Koike M 164

Koja K 928

Kolb D 493

Kolfschoten GM 921

Kollmannsberger C 458

Komatsu K 769
Kondo S 668

Konno R 1488

Kool M 366

Kornek G 454

Koskinen L-OD 826

Koukouraki S 1281

Koukourakis MI 219, 1281

Koumakis G 1128

Kreienberg R 1338

Kristjánsdóttir K 1715

Kroep JR 1069

Kröger N 874

Kroon BBR 1351

Kruczynski A 1516

Krüger W 874

Kuan A 91

Kubo N 215

Kuh D 964

Kulkarni S 91

Kuma K 1495

Kumai H 701

Kumar H 1425

Kundu SD 519

Kuninaka S 928

Kuper H 949, 1234

Kuriyama T 858

Kuroda S 1168

Kusiak I 1664

Kuwano H 609, 1033

Kvalheim G 1375

Kyrias G 1281

Kytölä S 1020

La Vecchia C 689, 952, 1238

Laake K 1650

Labianca R 573

Lacornerie T 642

Ladner RD 792

Lafitte J-J 1128

Laiho P 1015

Lakari E 880

Lallemand E 1623

Lamb J 487, 800

Lambin P 514

Landman-Parker J 1617

Langman MJS 550

Lankelma J 375

Larsen RH 1375

Larsson B 307

Larsson C 1020

Launonen V 1015

Laurence V 1480

Laylor R 1202

le Maho Y 1055

Leal JE 969 (L)

Leblanc T 1617

Lee AS-G 750

Lee BL 1360

Lee C 519

Lee J-W 1674

Lee SM 1405

Lee-MacAry AE 1202

Lefebvre JL 1594

Lefrere-Belda M-A 209

Leibovitz I 463

Leigh DA 729

Lejars O 973

Lejeune FJ 1351

Lennon A 421

Leo F 569
Leonard P 418 (BR)

Leonard RCF 98, 135

Leslie MD 863

Levack P 98

Leverger G 973, 1617

Levi F 689, 952

Levin VA 588

Lewis C 438

Li G 1468

Li L-Y 1154

Li X 50, 407

Lie SO 1124

Lilge L 1110

Lim CK 935

Lin S 1762

Lin VCL 319

Lin X 1360

Lindgren A 391

Lindhofer H 261

Linet M 692

Ling Y-Z 74

Lingenfelser T 458

Lingnau K 1192

Lippman ME 1154

Lipworth L 1255 (L)

Lissia A 1707

Liu A 1154

Liu D 1473

Liu F 1256 (L)

Livne PM 463

Lloyd D 1118 (BR)

Lo Cunsolo C 1295

Locatelli MC 707

Lodge AJ 498

Logan RFA 127

Logue JP 620

Lohner R 874

Loncaster JA 620

Long BJ 74

Longnecker MP 404

Lønning PE 1650

Looijenga LHJ 729

Loos M 1192

López-Michelena T 847

López-Soriano FJ 526

López-Soriano J 526

Los G 1047

Lotayef M 30

Lotze MT 506

Love SD 219

Loves WJP 1069

Luben R 121

Luboinski B 1594

Lucchi M 480

Lunec J 40

Luo C 1525

Lutz P 973

Lynch SF 1443

Lyng FM 1223

Lyng H 354

Ma I-F 1510

Macadam RCA 188

McAnulty RJ 650

McBride M 692

McClaren B 1612

McClay EF 16

McClay MET 16

McCrea PD 870

MacDonald N 279 (L)
McGee JO'D 750

McGown A 1599

McGuckin AG 40

Machin D 699 (L), 959

McIllmurray MB 447

McIntyre IG 992

McIntyre K 1405

McKendrick J 438

McKeown SR 1589

McKinney PA 127, 387

MacKinnon AC 941

McLeish R 1084

McLeskey SW 1154

McNutt M 112

McWilliam P 725

Maddau C 1462

Madsen EL 1256 (L)

Maehara Y 887, 986, 1168

Mælandsmo GM 743

Maeurer MJ 1192

Magdelenat H 1318

Maggi G 569

Magnani C 104

Magnenet P 642

Mah-Becherel MCM 346

Maisey NR 287

Maisonpierre PC 1154

Maitland NJ 1102

Maize JC 16

Makuuchi M 50

Malan A 1055

Malcolm AJ 40, 397

Malignant Melanoma

Cooperative Group 1351

Malik K 1583

Mancuso S 1762

Manfioletti G 1503

Mangili A 892

Manning J 473

Mannion EM 1432

Manusama ER 1176

Mao JH 566

Marandas P 1594

Marangoni E 514

Margison GP 69, 1702

Markham AF 188, 329

Marres HAM 674

Marsden B 602

Marti M-C 1637

Marty M 431, 577

Mason M 655, 1612

Massey A 146

Masters S 1268

Mathiot C 1454

Matsunaga T 1020

Matsuzawa Y 668

Matsuzuka F 1495

Matthay KK 1121

Matthews CS 270

Matthews E 519

Matthey B 252

Mauch C 1387

Maughan TS 447

Mead GM 1623

Mechelany-Corone C 715

Mechinaud F 973

Mehta J 91

Mehtali M 1454

Meijers-Heijboer EJ 384

Melamed R 1747 
Melanoma Cooperative Group 1707

Mendes R 853

Menon U 279 (L)

Merlin J-L 1380

Mertins S 817

Metcalf JS 16

Metzger R 473

Miano R 1432

Miccoli P 1412

Michaeli D 443

Michaelis J 692

Michetti G 573

Michon J 973

Middleton H 602

Mignard D 431

Mikaelsdóttir EK 1715

Millon R 1380

Millot F 973

Millward M 438

Milroy R 447

Min Y 1047

Minard V 973

Mineta H 775

Mirabelli D 104

Mirjolet J-F 1380

Misawa K 775

Misra V 998

Mitachi Y 141

Mitchell C 602

Mitchell H 1432

Mitchell P 91

Mittelman A 360

Mittler U 756

Miura K 775

Miura S 833

Miyamoto K 1209

Miyamoto S 1168

Miyauchi A 1495

Miyazaki M 887

Miyazaki Y 668

Miyoshi J 769

Mizukami T 1039

Mochizuki Y 1607

Mommen P 1128

Monks NR 267

Monroe L 16

Monson J 1425

Moonga BS 360

Moore J 63, 980

Morbidelli L 63

More L 397

Morel P 1637

Mori N 1026

Moriya T 858, 1488

Moriyama N 1394

Morris Jones P 602

Mothersill C 1223

Motoda H 1039

Mouret E 1318, 1480

MRC Testicular Tumour

Working Party 1623

Mucci LA 1234

Mueller J 1323

Mulatero C 1612

Mulder NH 184

Muller C 514

Muñoz N 1238

Muramatsu H 701

Muramatsu T 701
Murata K 769

Murphy MFG 1231

Murray PV 1418

Muskiet F 594

Muslumanoglu M 737

Mussi A 480

Mutgi AB 133 (L)

Mysliwietz J 261

Nagano H 701

Nagao K 858

Nagasue N 1394

Nagawa H 324

Nakahara T 1607

Nakamura H 769

Nakamura M 833

Nakamura S 164, 1681

Napier MP 1274

Naraghi A 1274

Narayanan S 1532

Nass SJ 1688

Negri E 689

Nehmé A 1360

Nejad-Sattari M 1367

Nekarda H 1323

Ness RB 404

Newell DR 267

Newton R 412

Ng S-C 83

Ng SW 498

Nicholl I 1643

Nicholson M 447

Nicolas A 1318

Nicolini A 1412

Ninane V 1128

Nischt R 1387

Nnane IP 74

Nocera M 715

Noci I 1722

Noferini D 1462

Noga SJ 1405

Noguchi T 215

Nogueira M 1139

Noma T 1026

Noppen C 204

Norman A 287

Normand C 561

Norton A 1418

Numico G 1437

Nyrén O 391, 949

O'Brien MER 853, 1418

O'Brien PH 16

O'Byrne KJ 219

Oda M 701

Oda S 986

O'Doherty MJ 863

O'Dwyer ST 1344

Oevermann K 583

Ogino T 775

Ögmundsdóttir HM 1715

Oh Y 756

Ohly KV 1405

Ohnishi K 1735

Ohnishi T 1735

Ohnishi Y 833

Ohno S 609, 1033

Ohtsu A 141

Ojima I 1762

Oka M 1026, 1209
Okamoto K 701, 833

Okamura K 1488

Okuyama T 1394

Ólafsdóttir K 1715

Old LJ 493

Olive PL 1525, 1740

Oliver RTD 1612, 1623

Olivotto M 1722

Ollivaud L 1243

Olsen JH 692

Olson E 1559

Olson J 588

Olson TA 1077

Ondrey FG 1367

Oosterhuis JW 729

Orr S 270

Orsetti B 1309

Osada T 324

Oshika Y 833

Osoba D 588

Österreicher C 454

Osterwalder B 22

Ota I 1735

Ott RJ 287

Oudart H 1055

Owens C 602

Oya M 626

Ozbilen S 737

Ozcelik H 737

Pääkkö P 880

Padhani A 287

Paesmans M 1128

Páez de la Cadena M 1139

Page WF 1231

Paglierani M 1722

Palangié T 1480

Pallestrini E 1437

Palmer J 1003, 1009

Palmer RD 853

Palmieri G 1707

Pan T-L 1096

Papelard H 719

Paridaens R 594

Parikka M 614

Parker L 397

Parkins CS 811

Parle-McDermott A 725

Passos-Coehlo J-L 1405

Pastorino U 569, 935

Patael Y 153

Patterson AV 219

Patterson LH 1589

Patzelt T 583

Paul J 426

Paus E 743

Peacock JH 650

Peak S 63

Pearson ADJ 40

Pellegriti G 715

Penz M 454

Perel Y 973, 1617

Perrin D 1516

Perrone F 707

Perusinghe N 177

Peters AM 684

Peters GJ 1069

Petersen G 1643

Peto J 1565

Petridou E 1234
Pettersson F 239

Petti AR 1295

Peyrat J-P 1380

Peyroulet MC 973

Phillips P 588

Piazza E 707

Pierga J-Y 1480

Pignatelli F 1762

Pinedo HM 921, 1069

Pinkerton CR 338

Pirastu M 1707

Pirmoazen N 1249

Pirtskhalaishvili G 506

Pistolesi F 480

Plantaz D 973

Planting ASTh 22

Platt-Higgins A 1473

Pleau JM 1454

Poletti P 573

Ponce-de-León S 969 (L)

Poon TCW 1330

Popov Z 209

Porteous M 1643

Portnoy M 1110

Potten CS 1344

Potter DM 914

Pouillart P 1454, 1480

Povey AC 69

Powell JJ 1443

Powles R 91

Powles T 91

Prados MD 588

Praeuer HW 473

Price P 281, 1581 (BR)

Price T 146

Priest K 853

Pritchard J 602

Pritchard-Jones K 177

Pronk LC 22

Propper DJ 219

Puhakka A 880

Pujol J-L 8

Punnonen K 1161

Quadri A 573

Quietzsch D 458

Quinn LS 526

Quinn M 1556

Quinn MJ 278 (L)

Raderer M 454

Radvanyi F 209

Rafnar T 1715

Ragnarsson GB 1715

Rahman I 941

Rahman N 177

Rajamäki A 1161

Raleigh JA 674, 1525

Raleigh SM 935

Ramakrishnan S 1077

Ramirez J 104

Ramon Y 463

Rampling R 588

Randimbison L 952

Ranson M 1599

Rao S 146

Rapley EA 177

Raton JA 847

Rauth AM 998

Read A 278 (L) 
Reed MWR 1061

Rees RC 1061

Reeve A 177

Reid M 397

Reigner B 22

Reis AC 1243

Renehan AG 1344

Reynolds CP 1121

Rialland X 973

Ricci I 1437

Richardson G 438

Rijken PFJW 674

Rindi G 952

Rindsfüser M 637

Rintoul R 941

Rischin D 438

Ristori E 1503

Rittling SR 156

Riva A 532, 1762

Rizvi I 1544

Robbiati SF 707

Roberts DD 298

Roberts I 1309

Roberts JT 1623

Robinson PA 188

Rochlitz C 1454

Rocmans P 569

Roder RJ 35

Rodríguez-Berrocal FJ 1139

Roelofs H 729

Rofstad EK 354

Rohner S 1637

Roman E 692

Romerio F 532

Ronco G 1462

Roovers RC 252

Rosati B 1722

Rosenberg R 35, 1323

Rosenfeld RG 756

Rosenne E 1630

Rosenthal AN 1287

Roshan MK 1249

Ross EL 1202

Ross JA 700 (L), 1443

Ross PJ 146

Rosso R 1437

Roth AD 1637

Roth JA 473

Roth S 1015

Rothman VL 298

Rotmensz N 1243

Rougier P 431

Rowlinson-Busza G 232, 684

Royer-Pokora B 177

Rubie H 973

Rudland P 1084

Rudland PS 1473

Ruiter DJ 1351

Rumi MAK 1394

Runnebaum IB 1338

Rustin GJS 1274

Ryan A 1287

Ryan C 853

Rylander E 307

S-1 Cooperative Colorectal Carcinoma Study Group 141

Sackett D 817

Saeki H 609

Sage RD 134 (L)
Sahin AA 870

Saidi F 1249

Säily M 880

Saini A 853

Saip P 737

Saito S 324

Saito T 1168

Sakata Y 141

Sakuma S 701

Sala E 121

Salehian P 1249

Salles B 514

Sällström J 307

Salminen E 1161

Salo T 614

Salom AV 1480

Salovaara R 1015

Salup RR 506

Samaritani E 480

Sancho-Garnier H 1594

Sandler DP 404

Sandor V 817

Sanguineti G 1437

Santelli A 1437

Sarela AI 188

Sasano H 1488

Sasieni P 561

Saso R 91

Sastre-Garau X 1318, 1454

Sathyamoorthy N 333

Sato H 1394

Sato S 1488

Sauroja I 1161

Sausville E 817

Sausville EA 1401

Savage P 980

Sawa N 833

Scambia G 1762

Schaison G 1617

Schally AV 906

Schey S 1268

Schlumberger M 715

Schmerber J 1128

Schmitt B 261

Schmitt E 1192

Schneider PM 473

Scholfield DP 443

Scholl S 1480

Schrader A 583

Schraffordt Koops H 184, 1351

Schroeder M 458

Schultz-Thater E 204

Schultze-Seemann W 637

Schulz WA 626

Schumacher V 177

Schurz H 782

Scott D 1136

Scott KA 1538

Scott L 800

Scott PAE 63

Screpanti I 1503

Sculier J-P 1128

Scurry JP 1659

Seabra L 1084

Seeghers M 594

Segnan N 1462

Selker RG 588

Senderowicz A 817

Seo Y-C 750

Seow-Choen F 750
Sepehr A 1249

Sethi T 941

Severi G 1243

Severson RK 700 (L)

Seymour CB 1223

Seynaeve C 384

Seynhaeve ALB 1176

Shakhar G 1630, 1747

Shakhar K 1630

Shalet SM 1344

Shamash J 1258 (L)

Shannon R 602

Shapiro W 588

Sharp L 127

Sharpe CR 112

Sharrard RM 1102

Sheer D 40

Sheffield E 69

Shelling A 177

Shen GH 196

Shepherd J 1003, 1009

Shi Y-Z 50

Shibata Y 324

Shimizu H 196

Shinohara H 1039

Shinomura Y 668

Shohtsu A 1607

Shouman T 30

Shtriker A 463

Shurin GV 506

Shurin MR 506

Sibson DR 1473

Sidi AA 463

Siebert R 743

Siersema PD 539

Signorello LB 391

Sikic BI 892

Silvester J 935

Simán SE 69

Simms MS 443

Simpson A 426

Singh R 1654

Skeen J 177

Skinner J 692

Skovlund E 1650

Skytthe A 1231

Smeland EB 743

Smid-Koopman E 246

Smith A 487

Smith AJ 366

Smith DR 761

Smith GL 1432

Smith IE 853, 1268, 1418

Smith J 438

Smith S 800

Snary D 1202

Söderström, K-O 1161

Soini Y 614, 880

Sommelet D 1617

Souberbielle BE 853

Souhami R 1771 (BR)

Soussi T 1418

South and West Regional Cancer

Organisation Tumour Panel for

Head and Neck Cancer 421

Spagnoli GC 204

Sparidans R 375

Sparreboom A 22

Spence A 588

Spinelli C 1412
Spreadborough A 1136

Spreckley K 992

Squiban P 1454

Staniforth JN 935

Stark DPH 1261

Steele F 935

Steinbauer M 1216

Stenning SP 1623

Stephan JL 973

Stephen R 1183

Stephens RJ 447

Stevens MFG 270

Stevenson AJ 329

Steward M 498

Stewart AFR 134 (L)

Stewart JSW 232, 684

Stewart PM 550

Stewart THM 134 (L)

Stockert E 493

Stoeltzing O 473

Stoppa-Lyonnet D 1318

Straatman H 1351

Strand A 307

Stratton MR 177

Strazzullo M 1707

Stromboni-Luboinski M 1594

Suchy B 1664

Suciu S 6, 1351

Suetsugu H 1394

Sugimachi K 141, 609, 887, 986, 1033

Sugisaki Y 196

Summerhayes M 1268

Sun B 906

Sunami E 324

Sundfør K 354

Suzuki I 775

Suzuki N 769

Suzuki T 1488

Swansbury GJ 91

Swerdlow AJ 964

Symonds P 566

Syrigos KN 232, 684

Szepeshazi K 906

Tabeta H 858

Tabrizi SN 1659

Taddei GL 1722

Taguchi T 141

Tahir SK 83

Takahashi A 1735

Takahashi I 986

Takahashi R 1039

Takahashi W 1607

Takano T 1495

Takayama T 50

Takebayashi S 775

Takeno S 215

Takiguchi Y 858

Talamini R 689, 1238

Talbot DC 219

Tamaoki T 1039

Tan PH 319

Tanaka Y 56

Tanda F 1707

Tang W-Y 761

Tangoku A 1026, 1209

Tartour E 1454

Tatematsu M 1681

Tatsuta M 769 
Tavani A 1238

Tavassoli M 1418

Taylor C 1565

Taylor CPF 40

Taylor M 219

Taylor R 602

Taylor-Papadimitriou J 1202

Te V-C 952

Teh B 1003, 1009

ten Hagen TLM 1176

Terlouw EM 539

Terracini B 104

Thatcher N 447

Theillet C 1309

Theodorsson E 171

Thews O 225

Thiery JP 209

Thiriaux J 1128

Thomassen J 1405

Thorban S 35

Thyss A 973

Tiebosch ATMG 184

Tighe O 725

Tilanus HW 539

Timmer-Bosscha H 184

Timothy AR 863

Tischler T 463

Tisdale MJ 56

Tobias JS 971 (BR)

Todorov PT 56

Toh Y 928, 1033

Tohari S 750

Toi M 164

Tokunaga T 833

Tolis C 1069

Tollenaar RAEM 719

Tominaga T 164

Tomozawa S 324

Toner G 438

Tonetti DA 782

Tonini GP 1295

Toniolo PG 1255 (L)

Topping K 1425

Tozer GM 662, 811

Treleaven J 91

Trichopoulos D 949, 1234

Trillet-Lenoir V 431

Trock BJ 1688

Tropé CG 354

Truchetet F 1243

Tsuchida T 833

Tsuno NH 324

Tsuruo T 324

Tuckwell N 853

Turkmen S 737

Turley H 63

Turuguet D 104

Tuszynski GP 298
Twelves C 22

Tynes T 692

Uchida Y 215

Uciechowski P 1664

Ueda Y 775

Ueno T 164

Ueyama Y 833

Uges DRA 594

United Kingdom Childhood

Cancer Study Investigators 1573

United Kingdom Children's Cancer Study Group 40, 602

United Kingdom Co-ordinating Committee 294

United Kingdom NSCLC Gemcitabine Group 447

Underwood M 426

Uri N 1696

Usel M 104

Uster PS 232, 684

Vaccarella S 1238

Vaganay-Juéry S 514

Vaidya JS 1769 (BR)

Vaittinen P 407

Valencak J 454

van Ballegooijen M 561

Van Buuren I 594

van Deemter L 366

van Dekken H 539

van den Akker-van Marie E 561

van den Hoogen FJA 674

van der Graaf WTA 594

van der Kogel AJ 674

Van der Pavert I 899

Van der Schueren B 899

van der Wall E 1405

van Eijk R 719

van Etten B 1176

Van Geel AN 569

van Geloof WL 1351

van Groeningen CJ 1069

van Hillegersberg R 539

van Muijen GNP 1351

van Oosterom AT 594

van Tiel ST 1176

Van Waes C 1367

Vantard M 544

Varcoe S 219

Varley JM 467

Vasey P 22

Vaughan M 980

Vaupel P 225

Vazina A 463

Veitia R 544

Verhoog LC 384
Verkasalo PK 692, 1231

Vernillet L 146

Verschoyle RD 935

Verweij J 22

Vick NA 588

Vidarsson H 1715

Vincent F 346

Vincent-Salomon A 1318

Vitale V 1437

Vliet Vlieland TPM 719

Vogelsang G 1405

Vogl FD 1338

von Eyben FE 1256 (L)

Voorn DA 1069

Vorbeck F 454

Vorwerk P 756

Vu P 1650

Vujanic GM 602

Wadsworth M 964

Walker M 1646

Walker SJ 959

Walter W 1192

Walters M 1003, 1009

Walz A 261

Wang T-M 1510

Wang TTY 333

Wanke E 1722

Ward TH 69

Warenius HM 1084

Warn RM 1147

Warren R 121

Watanabe M 609, 1033

Watanabe R 858

Waters C 941

Webb A 287, 853, 980

Webley SD 792

Wegerer S 473

Weiderpass E 949

Weirich A 177

Welch A 121

Weltin D 642

Wennerberg J 775

West CML 620, 1702

Westhoff C 1552

Wetterauer U 637

Wex H 756

Wheatley DN 800

White DJ 1659

Whitehouse A 329

Whiteman DC 1231

Wibault P 1594

Wielinga PR 375

Wilander E 307

Wild R 1077

Wilkinson PM 1623

Wilkinson RW 1202

Williamson B 493
Wilson BC 1110

Wilson CR 1702

Wilson GD 30

Wilson JHP 539

Wollenberg B 261

Wu Y 156

Wu-Wong JR 360

Wijffels KIEM 674

Yaacobi Y 463

Yajima A 1488

Yamamoto K 1026

Yamamura Y 1681

Yamaoka Y 1039

Yamazaki H 833

Yang C-S 1674

Yang T 519

Yang YM 360

Yano A 701

Yap W-M 761

Yarnold JR 650

Yasuda M 1033

Yasuda S 1607

Yazdanbod M 1249

Yazici H 737

Yazumi S 1039

Ye W 949

Yeh K-H 1510

Yeh S-H 1510

Yokoyama H 1096

Yoshida H 1209, 1495

Yoshida T 1039

Yoshikawa K 1039

Yoshimura K 1209

Yoshino S 1026, 1209

Yu J 1154

Yue N 588

Yule R 1565

Yung WKA 588

Zacharias C 1128

Zafrani B 1318

Zajac P 204

Zaknoen S 588

Zander AR 874

Zani M 1503

Zeelenberg I 1047

Zehavi M 463

Zeidler R 261

Zella D 532

Zhang H-T 63

Zhang Q 1360

Zhou J 1039

Zhu L 1255 (L)

Ziche M 63

Ziegler I 261

Zigrino P 1387 